\title{
Early Skin Tumor Detection from Microscopic Images through Image Processing
}

\author{
AYESHA AMIR SIDDIQI*, GHOUS BAKSH NAREJO**, AREEBA MEHMOOD KHAN**, AND \\ MASHAL TARIQ***
}

RECEIVED ON 04.10.2016 ACCEPTED ON 22.11.2016

\begin{abstract}
The research is done to provide appropriate detection technique for skin tumor detection. The work is done by using the image processing toolbox of MATLAB. Skin tumors are unwanted skin growth with different causes and varying extent of malignant cells. It is a syndrome in which skin cells mislay the ability to divide and grow normally. Early detection of tumor is the most important factor affecting the endurance of a patient. Studying the pattern of the skin cells is the fundamental problem in medical image analysis. The study of skin tumor has been of great interest to the researchers. DIP (Digital Image Processing) allows the use of much more complex algorithms for image processing, and hence, can offer both more sophisticated performance at simple task, and the implementation of methods which would be impossibly by analog means. It allows much wider range of algorithms to be applied to the input data and can avoid problems such as build up of noise and signal distortion during processing. The study shows that few works has been done on cellular scale for the images of skin. This research allows few checks for the early detection of skin tumor using microscopic images after testing and observing various algorithms. After analytical evaluation the result has been observed that the proposed checks are time efficient techniques and appropriate for the tumor detection. The algorithm applied provides promising results in lesser time with accuracy. The GUI (Graphical User Interface) that is generated for the algorithm makes the system user friendly.
\end{abstract}

Key Words: Skin, Tumor Detection, Microscopic Images, Cellular Level, Image Processing, Graphical User Interface.

\section{INTRODUCTION}

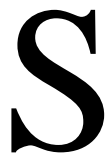
kin tumors are skin growth with different causes. There are three common types of skin cancer, basal cell carcinoma, malignant melanoma, squamous cell carcinoma. Basal cell carcinoma is grayish and like a pearl, few millimeter in size, appearing mostly on areas exposed to sun. It may spread to other organs as well. Squamous cell carcinoma is reddish in color from 5 millimeters to few centimeters in size, often with a central ulcer. Malignant melanoma is a growth of black or brown color, may appear anywhere on skin. All the tumor types may grow and penetrate into other organs.

Corresponding Author (E-Mail: 76ayesha@gmail.com)

* Department of Telecommunication Engineering, Dawood University of Engineering \& Technology, Karachi.

** Department of Electronics Engineering, NED University of Engineering \& Technology, Karachi.

*** $\quad$ Usman Institute of Technology, Karachi.

Mehran University Research Journal of Engineering \& Technology, Volume 36, No. 4, October, 2017 [p-ISSN: 0254-7821, e-ISSN: 2413-7219] 
Detection of tumor in minimum time is the most favorable condition to cure the patient [1-2]. It can be diagnosed by four major aspects which are asymmetry of tumor, border, color, diameter) known as the gross level detection. After the physical inspection of the tumor, the suspicious area (sample) is collected from the body to perform biopsy tests in the laboratory, after which the sample is examined under the microscope. Histopathology refers to the microscopic study of tissues in order to find out the symptom of disease. This procedure has its own limitation the major of which is time consumption by the medical professionals in order to detect the tumor. Sometimes a group of panel is required to study the case. The objective of the study is to find out the draw backs of offered procedures so far and recommend an automatic system. Transfer and interpret data through the use of appropriate tools and software like MATLAB through which the system is able to load, view and enhance the images using appropriate tools and application of proper algorithm in order to improve its quality.

Parolin et. al. [3] proposed a semi-automated technique for the diagnosis of melanoma through the analysis of dermatological descriptions. In the proposed approach a melanoma lesions are identified by a probabilistic classifier using Wavelet Transform [3]. Tasoulis et. al. [4] studied the classification of dermatological images by using clustering technique and image processing techniques for feature extraction through segmentation, border detection, color and texture processing for analysis of pigmented skin lesions in dermatological images. This method uses PCA (Principle Component Analysis).

Ma et. al. [5] made use of local fractals and multi-level wavelet analysis of boundary roughness of skin lesion. Boundary roughness is described using local fractals where as the wavelet packet is processed to give behavior of fractals at different frequency bands. Khaled, et. al. [6] proposed a hybrid approach in automatic segmentation and classification of skin lesion images that can be integrated with the system. This technique uses three different methods of automatic segmentations based on thresholding, morphological functions and active contours. Confer et. al. [7] studied the border detection of skin lesion images based on fuzzy C-mean thresholding consisting of two stages namely image pre-processing and image segmentations to locate skin. Border detection of skin tumor from digitized images was also found in previous work [8].

Kroemer et. al. [9] makes use of the mobile phones for skin tumor screening by utilizing the concept of teledermatology allowing the diagnosis of pigmented skin lesions. This system represents a filtering or triage system allowing a sensitive approach for the management of patients with emergent skin diseases. Usaj et. al. [10] proposed an automated cell counting tool for electro oration efficiency determination of attached cells in phase contrast images. This technique is based on the artificial neural network for counting nuclei. The technique integrates two image pre-processing steps: shading contrast and histogram equalization. The cell count was then automated by applying the Laplacian of Gaussian. Zheng et. al. [11] proposed a set of recursive equations for the fast calculation of divergence with an additional band to overcome the computational restrictions in realtime processing resulting in 90\% efficiency for skin tumor detection.

\section{MATERIALS AND METHOD}

\subsection{Materials}

The required data is comprised of specifically skin cases of patients suffering from basal cell carcinoma and malignant melanoma. The size of the images is $1280 \times 960$ pixels. The method is tested on Microsoft Windows 7 (Professional) with 2GB RAM and Intel(R) Core(TM) i3 CPUM380@2.53 GHz. The platform which is used for the analysis is MATLAB 7.0.

Mehran University Research Journal of Engineering \& Technology, Volume 36, No. 4, October, 2017 [p-ISSN: 0254-7821, e-ISSN: 2413-7219] 


\subsection{Correlation of Region of Interest with Reference Image}

In this method it is required to extract a mask image as in Figs. 1-2 or template typically much smaller than the original image. The best matches of the mask in the images in the original image are the locations of maximum values in the resulting correlation image. This method can be helpful in detecting different types of cells as well [11].

The methodology adopted for correlation of ROI (Region of Interest) with reference image is summarized in the following steps:

(i) Loading RGB (Red, Green Blue) image from the given cases.

(ii) Conversion of RGB values to Grayscale values.

(iii) Equalization of the grayscale image; mapping of grayscale values to new values so as to increase the contrast of the image.

(iv) ROI matrix manipulation.

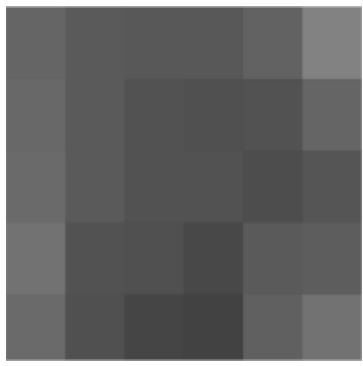

FIG. 1(a). MASK IMAGE(TEMPELATE)

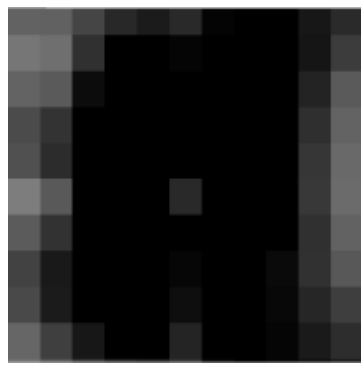

FIG. 1(b). LOCATION OF BEST MATCH (CORRELATED IMAGE) (v) Execution of 'for' loop followed by an 'if-else' condition; in order to determine the correlation of the ROI with the sample image.

(vi) The operation is repeated by sliding the recognized pattern over the image.

(vii) The tumor is detected at a point where ROI matrix is best matched to a desired level with the image.

\subsection{Calculation of Diameter of Cell}

This algorithm starts with the loading of RGB image from given cases and follows with the conversion of images to grayscale values. The next step is to equalize the image and map the grayscale values to new values so as to increase the contrast of the image .The global threshold level is adjusted so that it can be used to convert a grayscale image to a binary image. The boundaries of the cells as well as holes in the objects in the binary image are traced out and conversion of the label matrix to RGB values is done. The boundaries of the cells in the image are plotted. Calculation of the perimeter can be done through the difference between the adjacent elements of the cell matrix and approximating their derivative. Calculation of the diameter is done through perimeter. The calculation is based on pixel values. Tumor is detected by comparing the diameter of the cell to a desired level as shown in Fig. 3.

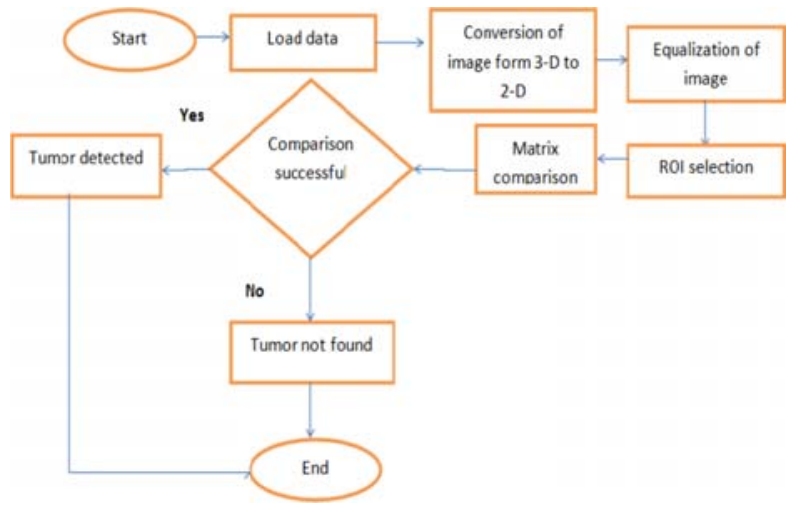

FIG. 2. FLOW CHART SHOWING STEPS FOR CORRELATION OF ROI WITH THE REFERENCE IMAGE

Mehran University Research Journal of Engineering \& Technology, Volume 36, No. 4, October, 2017 [p-ISSN: 0254-7821, e-ISSN: 2413-7219] 


\subsection{Pattern Recognition}

PCA (Principle Component Analysis) is used for identifying patterns in data, and expressing the data in such a way as to highlight their similarities and differences. The mean of the images is found out by first converting the mean image to grayscale image. Find the covariance of the grayscale image. Determination of six largest Eigen values from the Eigenvectors is done. The difference between the Eigen vector of the mean image and the Eigen vector of the individual grayscale image is necessary to detect tumor by comparing the result to a desired level (Fig. 4).

\section{RESULTS}

This paper provides a single platform to study the three aspects necessary to detect tumor. They are the intensity
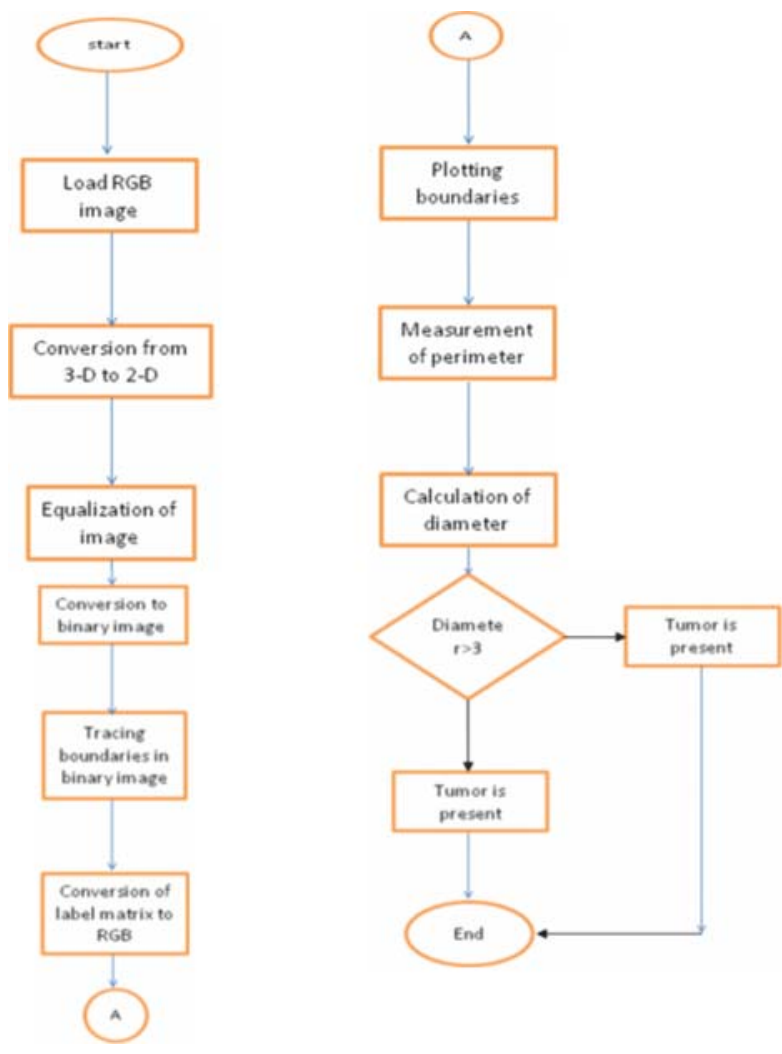

FIG. 3. FLOW CHART FOR THE CALCULATION OF DIAMETER OF THE CELL check Fig. 5; diameter check Fig. 6 and pattern recognition Fig. 7. User friendly GUI based algorithm has been introduced. The panel consists of three push buttons for intensity, diameter and pattern recognition, two static text boxes one panel to group all three push buttons and four axes to show the images. The original images and tumor detection by three different methods is shown in Figs. 5-7.
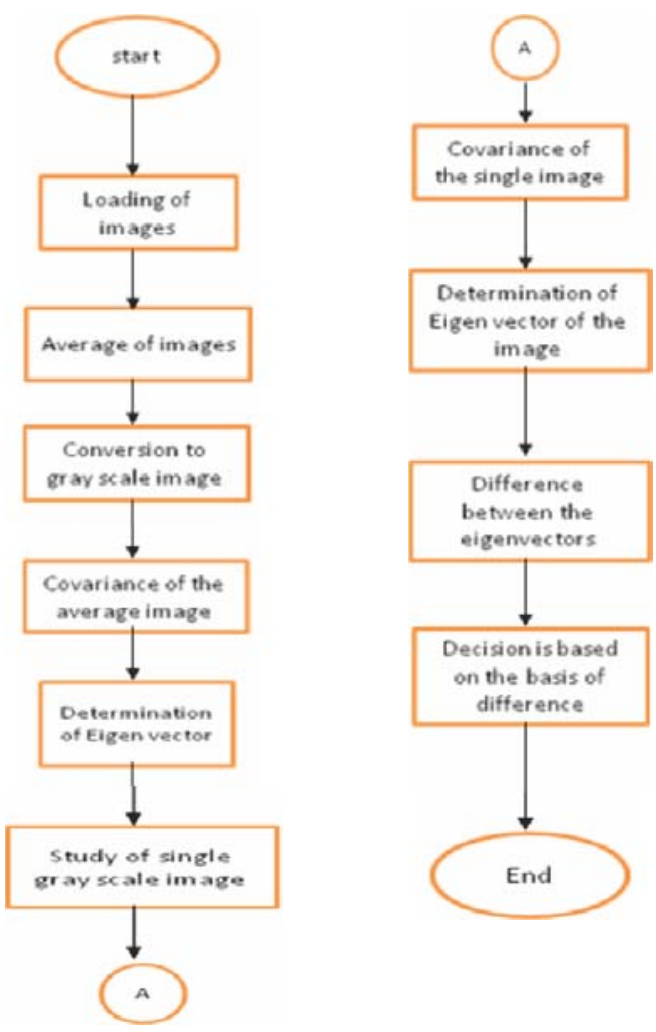

FIG. 4. FLOW CHART FOR PATTERN RECOGNITION

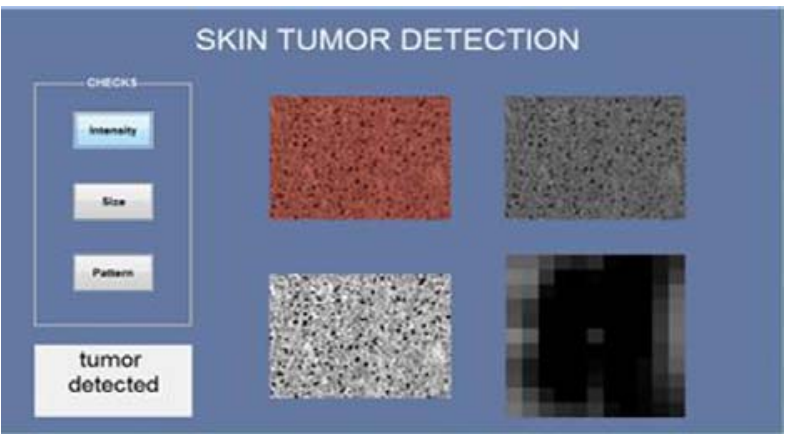

FIG. 5. GUI FOR INTENSITY CHECK 
The proposed algorithm is applied on a dataset of six cases of melanoma i.e. malignant melanoma and metastatic melanoma. The tumor is analyzed on the basis of the diameter of the cells by the radiologist. The accuracy table for this algorithm as compare to the radiologist study is shown in Table 1 the algorithm turns out to be $96.5 \%$ efficient.

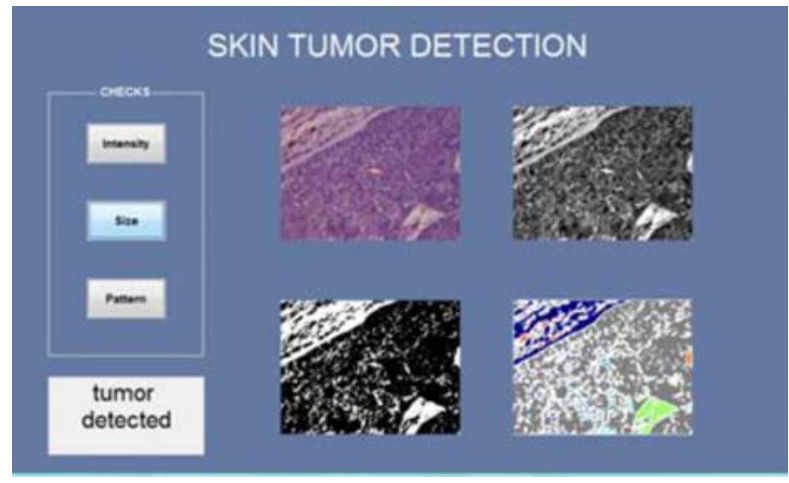

FIG. 6. GUI FOR DIAMETER CHECK

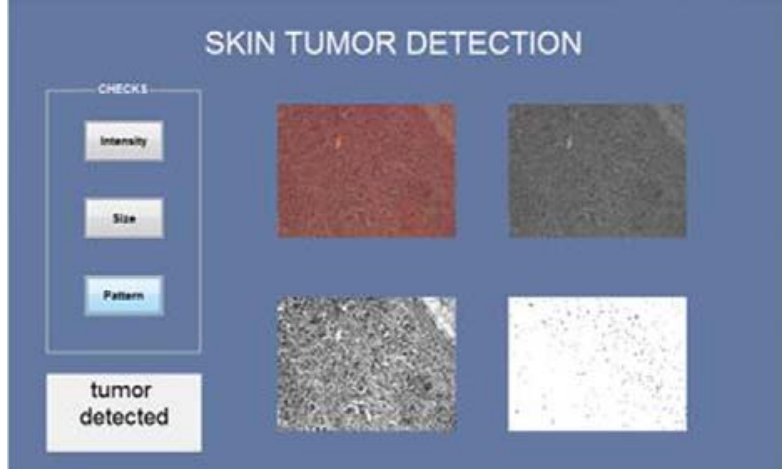

FIG. 7. GUI FOR PATTERN RECOGNITION

\section{CONCLUSION}

DIP, image processing in MATLAB and microscopic images of skin are studied. Different approaches were implemented after going through different algorithms. The primary problem is that the ROI is spread over the entire image along with the pigments. The three checks based on intensity, diameter and pattern recognition were tested. After the thorough study it has been found out that the intensity and diameter checks provide desirable and more accurate results as compare to pattern recognition check for melanoma. The proposed algorithm is applied on two cases of melanoma i.e. malignant melanoma and metastatic melanoma.

\section{FUTURE WORK}

The project can be extended to detect skin tumor in other types (i.e. basal cell carcinoma and squamous cell carcinoma). Further work is needed to be done for pattern recognition through eigenvectors with higher accuracy and lesser time.

\section{ACKNOWLEDGEMENT}

Authors would like to thank Dawood University of Engineering \& Technology, and NED University of Engineering \& Technology, Karachi, Pakistan, for providing immense support, guidance and help.

TABLE 1. RESULTS OBTAINED BASED ON THE DIAMETER OF THE TUMOROUS CELLS IN COMPARISON WITH THE RADIOLOGY RESULTS

\begin{tabular}{|c|c|c|c|c|}
\hline Cases & $\begin{array}{c}\text { Radiology Result } \\
(\mathrm{mm})\end{array}$ & $\begin{array}{c}\text { Algorithm Result } \\
(\mathrm{mm})\end{array}$ & $\begin{array}{c}\text { Difference } \\
(\mathrm{mm})\end{array}$ & $\begin{array}{c}\text { Accuracy } \\
(\%)\end{array}$ \\
\hline Case-1 & 4.02 & 4.16 & 0.14 & 97.00 \\
\hline Case-2 & 3.62 & 3.82 & 0.20 & 95.00 \\
\hline Case-3 & 3.98 & 4.16 & 0.18 & 96.00 \\
\hline Case-4 & 3.16 & 3.16 & 0.00 & 100.00 \\
\hline Case-5 & 4.62 & 4.89 & 0.27 & 94.00 \\
\hline Case-6 & 5.00 & 5.12 & 0.12 & 98.00 \\
\hline
\end{tabular}

Mehran University Research Journal of Engineering \& Technology, Volume 36, No. 4, October, 2017 [p-ISSN: 0254-7821, e-ISSN: 2413-7219] 


\section{REFERENCES}

[1] Mashal, T., Shehla, A., Ayesha, A., and Munawar, H., "Detection of Skin Cell Pattern from Effective Microscopic Images” The Environ Monitor - Journal of Engineering, Sciences \& Technology, Pakistan, Volume XV, pp. 12-17, [ISSN: 1684-8012] September-October 2015.

[2] Nassim, J., Kamran, K., Reinhard, G., Mohammad, S.1., Mohamad, J., and Fabrice, W., "Skull and Fontanel Segmentation from Neonatal CT Data by Model Based Variational Level Set Using Localized Coefficient”, IEEE Conference on Signal and Image Processing Applications, Malaysia, 16-18 November, 2011.

Parolin, A., Herzer, E., Jung, C.R., Univ, D., Vale, D.R.D., Sinos, S., and Leopoldo, B., "Semi-Automated Diagnosis of Melanoma through the Analysis of Dermatological Images”, $23^{\text {rd }}$ Conference on Graphics, Pattern and Images, 2010.

Tasoulis, S.K., Doukas, C.N., Maglogiannis, I., and Plagianakos, V.P., "Classification of Dermatological Images using Advanced Clustering Techniques”, Annual IEEE International Conference on Engineering in Medical and Biology Society, 2010,

Ma, L., Huang, K., Yan, J., Wu, K., and Zhu, L.,’Boundary Roughness Analysis of Skin Lesions Using Local $4^{\text {th }}$ International Conference on Fractals and Wavelet Transforms”, Bioinformatics and Biomedical Engineering, 2010.
[6] Khaled, T., and Nadra, B., "Automatic Segmentation and Classification of Skin Lesion Images", $2^{\text {nd }}$ International Conference on Distributed Frameworks for Multimedia Applications, 2006.

[7] Confer, S., "Border Detection of Skin Lesion Images Based on Fuzzy C-Means Thresholding”, 3 ${ }^{\text {rd }}$ International Conference on Genetic and Evolutionary Computing, 2009.

[8] Zhang, Z., Stoecker, V., and Moss, H., "Border Detection on Digitized Skin Tumor Images”, IEEE Transactions on Medical Imaging, Volume19, No. 11, pp. 1128-1143, August 6, 2002.

[9] Kroemer, S.1., Hofmann, W., Soyer, H., Koppitz, M., and Massone, C., "The Use of Mobile Phones for Skin Tumor Screening”, Medical Informatics Meets eHealth, Tagungsb and der eHealth, 2008.

[10] Usaj, M., Torkar, D., Kanduser, M., and Miklavcic, D. "Cell Counting Tool Parameters Optimization Approach for Electroporation Efficiency Determination of Attached Cells in Phase Contrast Images”, Journal of Microscopy, Volume 241, Part-3, pp. 303-314, 2011.

[11] Zheng, D., Myong, K., and Seong, G., "Band Selection of Hyperspectral Images for Automatic Detection of Poultry Skin Tumors", IEEE Transactions on Automation Science and Engineering, Volume 4, pp. 332-339, 2007. 